Original article

\title{
Childhood infections, vaccinations, and tonsillectomy and risk of first clinical diagnosis of CNS demyelination in the Ausimmune Study
}

\section{A.M. Hughes ${ }^{\mathrm{a}, \mathrm{b}}$, A.-L. Ponsonby ${ }^{\mathrm{c}, \mathrm{d}}$, K. Dear ${ }^{\mathrm{e}}$, T. Dwyer ${ }^{\mathrm{d}}$, B.V. Taylor ${ }^{\mathrm{f}}$, I. van $\operatorname{der}^{\text {Mei }}{ }^{\mathrm{f}}$, P.C. Valery ${ }^{g}$, Ausimmune Investigator Group, R.M. Lucas ${ }^{\mathrm{a}, \mathrm{h},}$}

${ }^{a}$ National Centre for Epidemiology and Population Health, The Australian National University, Canberra, Australian Capital Territory, Australia

b The Canberra Hospital, Canberra, Australia

${ }^{\mathrm{c}}$ The Florey Institute of Neuroscience and Mental Health, Melbourne, Australia

${ }^{\mathrm{d}}$ Murdoch Childrens Research Institute, University of Melbourne, Melbourne, Australia

${ }^{\mathrm{e}}$ School of Public Health, University of Adelaide, Adelaide, Australia

${ }^{\mathrm{f}}$ Menzies Institute for Medical Research, University of Tasmania, Hobart, Australia

${ }^{g}$ QIMR Berghofer Medical Research Institute, Brisbane, Australia

${ }^{\mathrm{h}}$ Centre for Ophthalmology and Visual Science, University of Western Australia, Perth, Australia

\section{A R T I C L E I N F O}

\section{Keywords:}

Multiple sclerosis

Infections

Vaccinations

Tonsillectomy

Case-control study

Demyelination

\begin{abstract}
A B S T R A C T
Background: The association between childhood vaccinations and infections and risk of multiple sclerosis is unclear; few studies have considered age at vaccination/infection.

Objective: To explore age-related associations between childhood vaccinations, infection and tonsillectomy and risk of a first clinical diagnosis of CNS demyelination.

Methods: Data on case $(n=275,76.6 \%$ female; mean age 38.6 years $)$ and age- and sex-matched control $(n=529)$ participants in an incident population-based case-control study included self-reported age at time of childhood vaccinations, infections, and tonsillectomy. Conditional logistic regression models were used to calculate adjusted odds ratios (AOR) and 95\% confidence intervals (CI).

Results: Poliomyelitis vaccination prior to school-age was associated with increased risk of a first clinical diagnosis of CNS demyelination (AOR $=2.60,95 \%$ CI 1.02-6.68), based on a very small unvaccinated reference group. Late (11-15 years) rubella vaccination (compared to none) was associated with lower odds of being a case $(\mathrm{AOR}=0.47,95 \% \mathrm{CI} 0.27-0.83)$. Past infectious mononucleosis at $11-15$ years (AOR $=2.84,95 \% \mathrm{CI} 1.0-7.57)$ and $16-20$ years $(\mathrm{AOR}=1.92,95 \% \mathrm{CI} 1.12-3.27)$ or tonsillectomy in adolescence $(11-15$ years: $\mathrm{AOR}=2.45$, 95\%CI 1.12-5.35), including after adjustment for IM, were associated with increased risk of a first clinical diagnosis of CNS demyelination.

Conclusions: Age at vaccination, infection or tonsillectomy may alter the risk of subsequent CNS demyelination. Failing to account for age effects may explain inconsistencies in past findings.
\end{abstract}

\section{Introduction}

Increasing incidence of multiple sclerosis (MS) over the past 50 years (Ribbons et al., 2017), changing geographic patterns (Ascherio and Munger, 2007), and a relatively low level of heritability (International Multiple Sclerosis Genetics Consortium 2018), indicate that environmental factors play an important role in the risk of developing MS. There is now compelling evidence for some of these environmental risk factors: low sun exposure and/or low vitamin D, smoking, adolescent obesity, and past infectious mononucleosis (IM)
(Waubant et al., 2019).

The evidence for a role of common childhood infections in risk of MS is unclear, with inconsistent findings from epidemiological studies. For example, recent studies have shown increased risk of MS with past measles (Abbasi et al., 2017, Shaygannejad et al., 2016) or mumps (Abbasi et al., 2017) as well as no evidence of an association (WendelHaga and Celius, 2017, Ahlgren et al., 2009, Bager et al., 2004). Few studies have considered age at infection, and these have also returned inconsistent results (Bager et al., 2004, Gronning et al., 1993, Compston et al., 1986, Bachmann and Kesselring, 1998, Hays, 1992,

\footnotetext{
* Corresponding author at: National Centre for Epidemiology and Population Health, Research School of Population Health, The Australian National University, Canberra 2600, Australia

E-mail address: robyn.lucas@anu.edu.au (R.M. Lucas).
} 
Hernan et al., 2001, Sullivan et al., 1984).

There are similar inconsistencies in relation to childhood vaccinations and risk of MS, including for age at vaccination. In a recent systematic review (Mailand and Frederiksen, 2017), most studies showed no association between past vaccination to measles, mumps, rubella or the combined measles-mumps-rubella (MMR) vaccine and MS risk. Nevertheless, one study showed increased risk of MS in association with past vaccination with MMR or rubella alone (Zorzon et al., 2003). Most studies have found no association with past vaccination for diphtheria or tetanus, but other studies, including meta-analyses, have found a protective effect for these vaccinations (DeStefano et al., 2003, Hernan et al., 2006, Farez and Correale, 2011). Zilber and colleagues found that MS patients were less likely than controls to have been vaccinated against polio, but this was statistically significant only for vaccination with the live attenuated vaccine (Zilber and Kahana, 1996).

Here, we tested whether the age at infection and other related variables, including vaccination and tonsillectomy history, affected the subsequent risk of initial diagnosis of central nervous system (CNS) demyelinating disease in a well-characterised Australian case-control study.

\section{Methods}

The Ausimmune Study is described in detail elsewhere (Lucas et al., 2007). Briefly, this was a multicentre case-control study recruiting participants from November 2003 to December 2006. Cases $(n=282)$ were aged 18-59 years and had an incident first clinical diagnosis of CNS demyelination, including presentation with a classic clinically isolated syndrome (CIS; $n=216$ ), or a first diagnosis of primary progressive MS $(n=18)$ or where, in retrospect, there had been a previous, undiagnosed, probable CIS $(n=48)$. Controls $(n=558)$ were randomly selected from the Australian Electoral Roll (compulsory registration for citizens aged $\geq 18$ years) and matched to cases (matching ratio varying from $1: 1$ to $4: 1$ ) on age (within 2 years), sex and study region.

Participants were mailed a questionnaire to self-report demographic information and smoking history, and (with the assistance of parents if necessary, or health records where available) vaccination and infection history, including:

- whether or not they had received recommended early childhood vaccinations (diphtheria, pertussis, tetanus and poliomyelitis) on or before starting school;

- whether they had been vaccinated for, and the age-group (0-5, 6-10, 11-15 years) of the last vaccination (if multiple), for measles, mumps, rubella, smallpox, tuberculosis, and 'other' (free text for name of infectious disease).

- history of, and age at $(0-5,6-10,11-15,16-20,>20$ years), the following infections (first infection only if multiple): chickenpox (varicella zoster), measles, rubella, mumps, whooping cough (pertussis), cold sores (herpes labialis), infectious mononucleosis (IM), and school sores (impetigo).

- whether they had had a tonsillectomy and the age that this occurred.

At a face-to-face interview, case participants were asked whether they thought that infection was an important cause of their illness and blood was taken by venepuncture; serum was aliquoted and stored at $-80^{\circ} \mathrm{C}$ until the completion of data collection. Serum 25 -hydroxyvitamin D $(25(\mathrm{OH}) \mathrm{D})$ concentration was measured by liquid chromatography tandem mass spectrometry, as previously reported (Lucas et al., 2011).

\subsection{Statistical analysis}

\subsubsection{Data management}

Where a positive history of infection, vaccination or tonsillectomy was indicated, but age information was missing, age was coded as 'unspecified'. We categorized infections, vaccinations and tonsillectomy as either 'Early' ( $\leq 10$ years) or 'Late' ( $>10$ years of age). Most infections occurred at ages $6-10$ years $(n=622 ; 43 \%)$; the majority of the vaccinations were given at ages $11-15$ years $(n=452 ; 44 \%)$.

\subsubsection{Data analysis}

Continuous data were described using the mean and standard deviation (SD) or median and interquartile range (IQR), according to whether they appeared normally distributed or not, respectively. Categorical data were described using the number and proportion (\%) in each category. Smoking was calculated as total number of years that participants smoked, subtracting periods of cessation. We deseasonalised the 25(OH)D data, as previously described, based on the seasonal variation in the control samples (Lucas et al., 2011).

We used conditional logistic regression models to test the association between infections, vaccinations and tonsillectomy (all yes vs. no) and being a case with a first clinical diagnosis of CNS demyelination, estimating odds ratios (ORs) with $95 \%$ confidence intervals $(95 \% \mathrm{CI}$ ) and $p$ values, with and without adjustment for possible confounding by highest level of education, total years of smoking and 25(OH)D concentration. We also tested the risk of a first clinical diagnosis of CNS demyelination in association with infections, vaccinations, and tonsillectomy for different 5-year age groups, as well as categorised as 'Early' or 'Late' (see above).

Finally, we tested the effect on risk of a first clinical diagnosis of CNS demyelination of past IM and a second specific infection or tonsillectomy. We tested for effect modification by including a multiplicative term in the conditional logistic regression model. We next used a $2 \times 2$ factorial design to test the two "main effects"; we used the ic command in STATA to estimate the relative excess risk due to interaction (RERI), attributable proportion (AP) and Synergy Index (SI), to examine possible additive interaction.

We undertook a sensitivity analysis testing the major findings in the subgroup of cases who did not think that infection was an important cause of their illness and their matched controls, to rule out that any associations that were found were biased by belief that infection was an important cause of the participant's illness.

Participants with missing data on factors of interest were excluded from those specific analyses. Tests for linear trend across categorical variables were conducted by replacing the categorical predictor with a single predictor, taking category rank scores. All analyses were undertaken using STATA (Version 14.0; STATACorp LP, College Station, TX, USA). We did not adjust for multiple comparisons, as, although this reduces the risk of false positives, it increases the risk of false negatives (Rothman, 1990), particularly with the limited sample size available for some subgroup analyses. Rather than emphasising an arbitrary significance threshold, we present $p$-values as continuous estimates of the compatibility of the results with null hypotheses, as well as the $95 \%$ confidence intervals (CI) so that the reader is able to evaluate the effect of sample size on associations (Greenland et al., 2016).

The Ausimmune Study was approved by nine regional Human Research Ethics Committees. All participants gave written informed consent.

\section{Results}

The Ausimmune Study recruited 282 cases (91\% of those notified to the study) with a first clinical diagnosis of CNS demyelination and 558 population controls ( $60 \%$ of those contacted). Data on infections and/or vaccinations were available on $n=275$ cases and $n=529$ age- and sex-matched controls. Characteristics of these participants are shown in Table 1.

\subsection{Vaccinations of early childhood}

Most study participants (cases 86.5\%; controls 84.0\%) reported 
Table 1

Characteristics of case $(n=275)$ and control $(n=529)$ participants in the Ausimmune Study with data on childhood vaccinations, infections, and tonsillectomy.

\begin{tabular}{lll}
\hline & Case & Control \\
\hline Age [mean (SD)] years & $38.6(9.6)$ & $39.8(9.6)$ \\
Sex, [n (\%)] & $65(23.6)$ & $119(22.5)$ \\
$\quad$ Male & $210(76.4)$ & $410(77.5)$ \\
$\quad$ Female & $79(25.6)$ & $171(32.2)$ \\
Education, [n (\%)] & $136(49.4)$ & $220(41.6)$ \\
$\quad$ Year 10 or less & $68(24.8)$ & $138(26.1)$ \\
$\quad$ Year 12 + TAFE & $6.0(0-19.0)$ & $1.6(0-15.0)$ \\
$\quad$ University & $76.3(30.4)$ & $82.0(30.4)$ \\
Smoking [median(Q1, Q3)] years & & 0.06 \\
Serum 25(OH)D (mean(SD) nmol/L & & 0.04 \\
Cases only & $89(33.0)$ & $181(67.0)$ \\
Infection a likely cause of illness & & \\
$\quad$ Yes & & \\
No & &
\end{tabular}

TAFE: technical and further education; Q1: 25th percentile; Q3: 75th percentile; SD: standard deviation

receiving all four recommended early childhood vaccinations before or on starting school. We found no association with risk of a first clinical diagnosis of CNS demyelination for diphtheria, pertussis or tetanus vaccination (Table 2). There was a borderline significant increase in risk of a first clinical diagnosis of CNS demyelination in association with poliomyelitis vaccination in early childhood.

\subsection{Vaccinations prior to age 16}

Ever versus never. There were no significant differences in the frequencies of self-reported measles, mumps, rubella, smallpox or tuberculosis vaccination between the case and control groups, and no significant associations with risk of a first clinical diagnosis of CNS demyelination in the adjusted analyses (Supplementary Table 1).

Age at vaccination. Overall, age at vaccination against measles, mumps, smallpox, or tuberculosis was not significantly associated with risk of a first clinical diagnosis of CNS demyelination (Table 3). For rubella, vaccination at 11-15 years was associated with a reduced risk of a first clinical diagnosis of CNS demyelination and there was a significant protective trend for vaccination at a later age. There was some evidence to support a reduced risk of a first clinical diagnosis of CNS demyelination in association with older age at vaccination for smallpox (11-15 years) and younger age at vaccination for tuberculosis (0-5 years), compared to never being vaccinated for these disorders ( $p=0.06$ and $p=0.08$, respectively).

\subsection{1. 'Early' versus 'Late' vaccination}

'Late' vaccination against rubella was associated with a statistically significant lower risk of a first clinical diagnosis of CNS demyelination in comparison to both 'No vaccination' (AOR $=0.74,95 \% \mathrm{CI} 0.55-0.99$, $p=0.04$ ) and 'Early vaccination' (AOR $=0.36,95 \%$ CI $0.15-0.85$, $p=0.02)$. In the subgroup of individuals with no reported history of rubella infection ( $n=155$ cases; $n=319$ controls), the protective effect of 'Late' rubella vaccination was preserved for both the comparison with 'No vaccination' (AOR $=0.33$ (95\% CI $0.14-0.78$, $p=0.01$ ) and that with 'Early vaccination' (AOR $=0.31(95 \% \mathrm{CI}$ $0.09-1.06, p=0.06$ ). There was no association between 'Early' or 'Late' vaccination and risk of a first clinical diagnosis of CNS demyelination for measles, mumps, smallpox, or tuberculosis (Supplementary Table 1).

\subsection{History of infections}

Ever versus never. The distribution of infections and age at infection for cases and controls are shown in Table 4 and Supplementary Table 2. When infection history was assessed on an ever/never basis, only a history of past IM was associated with (increased) odds of a first clinical diagnosis of CNS demyelination (AOR $=2.04$, 95\%CI 1.39-3.00, $p<0.0001$ ) (Supplementary Table 2).

Age at infection. Table 4 shows the results of the analysis of age at infection and association with risk of a first clinical diagnosis of CNS demyelination. Overall, there was no significant association between age at diagnosis of chicken pox, measles, mumps, pertussis, or impetigo and risk of a first clinical diagnosis of CNS demyelination. Cases were significantly more likely than controls to report IM at 11-15 years and at $16-20$ years.

\subsection{1. 'Early' versus 'Late' infection}

'Late' IM (AOR $=1.43$, 95\%CI 1.17-1.75, $p<0.0001$ ), but not 'Early IM' (AOR $=1.54,95 \%$ CI $0.23-10.47$ ) compared to no IM was associated with a significant increase in the odds of a first clinical diagnosis of CNS demyelination. We found no evidence of an association with risk of a first clinical diagnosis of CNS demyelination for 'Early' or 'Late' infection with chicken pox, measles, mumps, pertussis, impetigo, rubella, or cold sores (Supplementary Table 2).

Table 2

The association between early childhood vaccination history, received on or before school, and a first clinical diagnosis of CNS demyelination

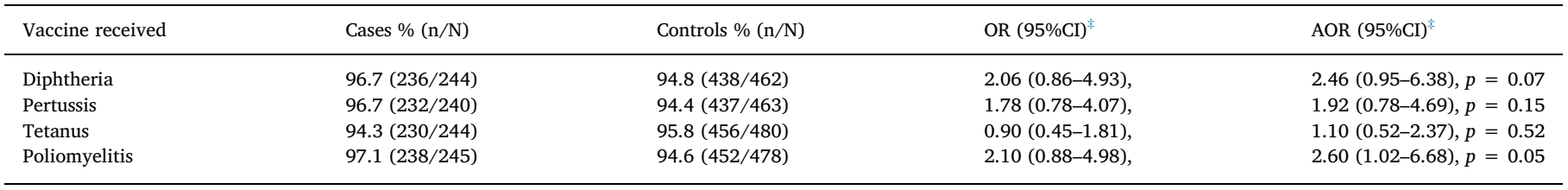

CNS: central nervous system demyelination, OR: odds ratio, CI: confidence interval

Numbers not adding to total sample size reflect a 'don't know' response to vaccination (as opposed to a missing value)

*Odds ratio adjusted for highest level of education, total years smoked and deseasonalied serum 25-hydroxyvitamin D (25OHD) level

$*$ Individuals without vaccination as reference category $(\mathrm{OR}=1.0)$ 
Table 3

The association between age at vaccination and a first clinical diagnosis of CNS demyelination

\begin{tabular}{|c|c|c|c|c|c|}
\hline Age at vaccination (years) & Cases \% (n/N) & Controls \% $(n / N)$ & OR $(95 \% \mathrm{CI})$ & $\mathrm{AOR}^{*}(95 \% \mathrm{CI})$ & $p$ \\
\hline \multicolumn{6}{|c|}{ Measles } \\
\hline Never & $35.4(74 / 209)$ & $37.6(157 / 418)$ & 1.00 (Reference) & 1.00 (Reference) & \\
\hline $0-5$ & $25.4(53 / 209)$ & $23.7(99 / 418)$ & $1.33(0.78-2.26)$ & $1.17(0.67-2.02)$ & 0.58 \\
\hline $6-10$ & $13.4(28 / 209)$ & $12.7(53 / 418)$ & $1.22(0.65-2.31)$ & $1.09(0.55-2.15)$ & 0.81 \\
\hline $11-15$ & $11.0(23 / 209)$ & $6.5(27 / 418)$ & $1.72(0.76-3.88)$ & $1.41(0.60-3.34)$ & 0.43 \\
\hline Test of trend & & & & & $0.49^{\dagger}$ \\
\hline Unspecified & $14.8(31 / 209)$ & $19.6(82 / 418)$ & & & \\
\hline \multicolumn{6}{|c|}{ Mumps } \\
\hline Never & $56.3(116 / 206)$ & $55.3(221 / 400)$ & 1.00 (Reference) & 1.00 (Reference) & \\
\hline $0-5$ & $13.1(27 / 206)$ & $13.3(53 / 400)$ & $0.92(0.49-1.72)$ & $0.87(0.45-1.69)$ & 0.68 \\
\hline $6-10$ & $12.1(25 / 206)$ & $9.8(39 / 400)$ & $1.22(0.65-2.29)$ & $1.18(0.60-2.31)$ & 0.64 \\
\hline $11-15$ & $7.8(16 / 208)$ & $5.8(23 / 400)$ & $0.86(0.37-2.03)$ & $0.71(0.29-1.74)$ & 0.46 \\
\hline Test of trend & & & & & 0.78 \\
\hline Unspecified & $10.7(22 / 206)$ & $16.0(64 / 400)$ & & & \\
\hline \multicolumn{6}{|c|}{ Rubella } \\
\hline Never & $26.8(60 / 224)$ & $23.5(103 / 438)$ & 1.00 (Reference) & 1.00 (Reference) & \\
\hline $0-5$ & $3.1(7 / 224)$ & $3.7(16 / 438)$ & $0.67(0.23-1.91)$ & $0.56(0.19-1.66)$ & 0.29 \\
\hline $6-10$ & $6.3(14 / 224)$ & $4.6(20 / 438)$ & $1.47(0.56-3.80)$ & $1.85(0.62-5.49)$ & 0.27 \\
\hline $11-15$ & $54.5(122 / 224)$ & $58.2(255 / 438)$ & $0.57(0.34-0.97)$ & $0.47(0.27-0.83)$ & 0.009 \\
\hline Test of trend & & & & & $0.01^{\dagger}$ \\
\hline Unspecified & $9.4(21 / 224)$ & $10.1(44 / 438)$ & & & \\
\hline \multicolumn{6}{|c|}{ Smallpox } \\
\hline Never & $71.0(157 / 221)$ & $65.8(262 / 398)$ & 1.00 (Reference) & 1.00 (Reference) & \\
\hline $0-5$ & $9.5(21 / 221)$ & $7.0(28 / 398)$ & $1.16(0.60-2.23)$ & $1.08(0.54-2.17)$ & 0.83 \\
\hline $6-10$ & $7.7(17 / 221)$ & $10.3(41 / 398)$ & $0.80(0.40-1.59)$ & $1.01(0.49-2.08)$ & 0.98 \\
\hline $11-15$ & $3.6(8 / 221)$ & $7.3(29 / 398)$ & $0.46(0.19-1.09)$ & $0.42(0.17-1.02)$ & 0.06 \\
\hline Test of trend & & & & & $0.15^{\dagger}$ \\
\hline Unspecified & $8.1(18 / 221)$ & $9.6(38 / 398)$ & & & \\
\hline \multicolumn{6}{|c|}{ Tuberculosis } \\
\hline Never & $57.8(119 / 206)$ & $56.2(230 / 409)$ & 1.00 (Reference) & 1.00 (Reference) & \\
\hline $0-5$ & $2.9(6 / 206)$ & $5.4(22 / 409)$ & $0.41(0.14-1.16)$ & $0.37(0.12-1.11)$ & 0.08 \\
\hline $6-10$ & $5.8(12 / 206)$ & $5.6(23 / 409)$ & $1.22(0.51-2.92)$ & $1.17(0.47-2.92)$ & 0.74 \\
\hline $11-15$ & $24.3(50 / 206)$ & $23.7(97 / 409)$ & $1.01(0.62-1.66)$ & $0.99(0.59-1.68)$ & 0.98 \\
\hline Test of trend & & & & & $0.87^{\dagger}$ \\
\hline Unspecified & $9.2(19 / 206)$ & $9.1(37 / 409)$ & & & \\
\hline
\end{tabular}

CNS: central nervous system demyelination, OR: odds ratio, CI: confidence interval

*Odds ratio adjusted for highest level of education, smoking history and serum 25-hydroxyvitamin D (25OHD) level

$\$$ Positive history of vaccination, but age received not specified

${ }^{\dagger} P$ value for a homogeneity test by which we test for a differential effect of vaccination at different ages within the age span $0-15$ years

\section{Tonsillectomy}

Having had a tonsillectomy (31\% cases; $25 \%$ controls) was associated with increased risk of a first clinical diagnosis of CNS demyelination (Table 5). In the subsample with data on tonsillectomy and history of IM, there was little change in the effect estimate for tonsillectomy (AOR $=1.42,95 \% \mathrm{CI} 0.96-2.10)$ after additional adjustment for past history of IM (AOR $=1.40,95 \%$ CI 0.94-2.08). In addition, there was no evidence of an association between IM and subsequent tonsillectomy among controls $(p=0.52)$. The adverse association between tonsillectomy and odds of a first clinical diagnosis of CNS demyelination was strongest (and only statistically significant) for the 11-15 years age group (Table 5). There was no significant association between 'Late' or 'Early' tonsillectomy and risk of a first clinical diagnosis of CNS demyelination.

\subsection{Infectious mononucleosis and a second specific infection or tonsillectomy}

History of IM was consistently associated with increased risk of a first clinical diagnosis of CNS demyelination, with or without a specific second infection (Supplementary Table 4). We found no evidence of multiplicative or additive interaction between a history of IM and also reporting a specific other infection. Similar analyses in relation to vaccination were uninformative due to the small sample size for the IM +/unvaccinated subgroups (data not shown).

\subsection{Sensitivity analysis}

Including only the 181 cases who stated that infection was a 'possible cause, not very important', 'not a possible cause', or 'don't know' (as opposed to those who thought infection was likely to be an important cause) and their matched controls $(n=335)$, the findings and effect sizes were very similar to the main results (results not shown).

\section{Discussion}

In this population-based incident case-control study of childhood vaccinations and infections in relation to risk of a first clinical diagnosis of CNS demyelination, the strongest and most consistent finding was increased risk in association with past history of infectious mononucleosis, particularly occurring during adolescence and older. A history of tonsillectomy was an independent risk factor for a first clinical diagnosis of CNS demyelination, with tonsillectomy at age 11-15 years the primary driver of this effect. Although there was an apparent increase in risk of a first clinical diagnosis of CNS demyelination in association with vaccination against poliomyelitis on or before starting school, this was based on a small sample size in the reference group (no vaccination), and so must be treated with caution. We found a lower risk of a subsequent first clinical diagnosis of CNS demyelination with late rubella vaccination (after age 10 years) compared to no vaccination or early vaccination. There was little evidence that past infections other than IM were associated with risk of a first clinical diagnosis of CNS demyelination. 
Table 4

Age at diagnosis of infections and risk of a first clinical diagnosis of CNS demyelination

\begin{tabular}{|c|c|c|c|c|c|}
\hline Age at diagnosis (years) & Cases \% $(n / N)$ & Controls \% $(n / N)$ & OR $(95 \% \mathrm{CI})$ & $\mathrm{AOR}^{*}(95 \% \mathrm{CI})$ & $\mathrm{p}$ \\
\hline \multicolumn{6}{|c|}{ Chicken pox } \\
\hline Never & $13.0(33 / 254)$ & $15.0(75 / 501)$ & 1.00 (Reference) & 1.00 (Reference) & \\
\hline $0-5$ & $18.9(48 / 254)$ & $19.8(99 / 501)$ & $1.06(0.60-1.86)$ & $1.27(0.68-2.35)$ & 0.45 \\
\hline $6-10$ & $41.7(106 / 254)$ & $41.5(208 / 501)$ & $1.08(0.65-1.77)$ & $1.17(0.68-2.01)$ & 0.57 \\
\hline $11-15$ & $10.6(27 / 254)$ & $9.6(48 / 501)$ & $1.20(0.62-2.31)$ & $1.30(0.65-2.63)$ & 0.46 \\
\hline $16-20$ & $2.4(6 / 254)$ & $3.4(17 / 501)$ & $0.78(0.27-2.19)$ & $0.85(0.28-2.55)$ & 0.77 \\
\hline$>20$ & $5.5(14 / 254)$ & $4.6(23 / 501)$ & $1.73(0.74-4.05)$ & $2.08(0.82-5.25)$ & 0.12 \\
\hline Test of trend & & & & & $p=0.31^{\dagger}$ \\
\hline Unspecified & $7.9(20 / 254)$ & $6.2(31 / 501)$ & & & \\
\hline \multicolumn{6}{|c|}{ Measles } \\
\hline Never & $37.5(88 / 235)$ & $39.2(183 / 467)$ & 1.00 (Reference) & 1.00 (Reference) & \\
\hline $0-5$ & $16.6(39 / 235)$ & $18.4(86 / 467)$ & $1.12(0.66-1.90)$ & $1.14(0.64-2.02)$ & 0.66 \\
\hline $6-10$ & $32.8(77 / 235)$ & $31.9(149 / 467)$ & $0.92(0.59-1.42)$ & $0.93(0.58-1.48)$ & 0.75 \\
\hline $11-15$ & $3.0(7 / 235)$ & $2.8(13 / 467)$ & $1.36(0.45-4.13)$ & $2.45(0.67-8.92)$ & 0.18 \\
\hline $16-20$ & $0.4(1 / 235)$ & $0.6(3 / 467)$ & $0.61(0.05-7.01)$ & $0.81(0.07-9.40)$ & 0.87 \\
\hline$>20$ & $0.4(1 / 235)$ & $0.4(1 / 467)$ & $2.52(0.15-41.66)$ & $2.97(0.18-50.19)$ & 0.45 \\
\hline Test of trend & & & & & $p=0.75^{\dagger}$ \\
\hline Unspecified & $9.4(22 / 235)$ & $6.9(32 / 467)$ & & & \\
\hline \multicolumn{6}{|c|}{ Rubella } \\
\hline Never & $69.8(155 / 222)$ & $71.5(319 / 446)$ & 1.00 (Reference) & 1.00 (Reference) & \\
\hline $0-5$ & $8.6(19 / 222)$ & $7.2(32 / 446)$ & $1.40(0.73-2.68)$ & $1.52(0.76-3.03)$ & 0.23 \\
\hline $6-10$ & $9.5(21 / 222)$ & $11.7(52 / 446)$ & $0.86(0.48-1.55)$ & $0.81(0.44-1.50)$ & 0.50 \\
\hline $11-15$ & $6.3(14 / 222)$ & $3.1(14 / 446)$ & $2.95(1.08-6.97)$ & $3.00(0.94-9.58)$ & 0.06 \\
\hline $16-20$ & $1.4(3 / 222)$ & $2.2(10 / 446)$ & $0.71(0.18-2.74)$ & $0.45(0.09-2.30)$ & 0.34 \\
\hline$>20$ & $1.4(3 / 222)$ & $0.9(4 / 446)$ & $3.11(0.27-35.74)$ & $3.17(0.25-40.00)$ & 0.37 \\
\hline Test of trend & & & & & $p=0.63^{\dagger}$ \\
\hline Unspecified & $3.2(7 / 222)$ & $3.4(15 / 446)$ & & & \\
\hline \multicolumn{6}{|c|}{ Mumps } \\
\hline Never & $53.1(128 / 241)$ & $54.0(252 / 467)$ & 1.00 (Reference) & 1.00 (Reference) & \\
\hline $0-5$ & $10.0(24 / 241)$ & $8.1(38 / 467)$ & $1.54(0.84-2.81)$ & $1.95(1.00-3.81)$ & 0.05 \\
\hline $6-10$ & $26.6(64 / 241)$ & $27.6(129 / 467)$ & $0.90(0.58-1.38)$ & $0.96(0.61-1.52)$ & 0.87 \\
\hline $11-15$ & $5.0(12 / 241)$ & $4.9(23 / 467)$ & $0.98(0.43-2.23)$ & $0.98(0.42-2.31)$ & 0.97 \\
\hline $16-20$ & $0.4(1 / 241)$ & $0.6(3 / 467)$ & $0.60(0.06-6.12)$ & $0.78(0.07-8.38)$ & 0.84 \\
\hline$>20$ & $0.4(1 / 241)$ & $1.5(7 / 467)$ & $0.24(0.03-2.10)$ & $0.36(0.04-3.60)$ & 0.39 \\
\hline Test of trend & & & & & $p=0.75^{\dagger}$ \\
\hline Unspecified + & $4.6(11 / 241)$ & $3.2(15 / 467)$ & & & \\
\hline \multicolumn{6}{|c|}{ Pertussis } \\
\hline Never & $91.9(225 / 245)$ & $91.7(464 / 483)$ & 1.00 (Reference) & 1.00 (Reference) & \\
\hline $0-5$ & $4.1(10 / 245)$ & $3.1(15 / 483)$ & $1.51(0.64-3.59)$ & $1.57(0.64-3.87)$ & 0.33 \\
\hline $6-10$ & $1.6(4 / 245)$ & $1.5(7 / 483)$ & $0.92(0.26-3.23)$ & $1.12(0.29-4.35)$ & 0.88 \\
\hline $11-15$ & $1.6(5 / 245)$ & $3.3(5 / 483)$ & $0.55(0.18-1.73)$ & $0.36(0.10-1.30)$ & 0.12 \\
\hline $16-20$ & $0.0(0 / 245)$ & $0.0(0 / 483)$ & -- & & - \\
\hline$>20$ & $0.0(0 / 245)$ & $0.0(0 / 483)$ & & & \\
\hline Test of trend & & & & & $p=0.32^{\dagger}$ \\
\hline Unspecified & $0.4(1 / 245)$ & $0.4(2 / 483)$ & & & \\
\hline \multicolumn{6}{|c|}{ Cold sores } \\
\hline Never & $59.1(159 / 269)$ & $55.4(287 / 518)$ & 1.00 (Reference) & 1.00 (Reference) & \\
\hline $0-5$ & $1.1(3 / 269)$ & $1.5(8 / 518)$ & $0.49(0.10-2.35)$ & $0.44(0.09-2.25)$ & 0.33 \\
\hline $6-10$ & $6.7(18 / 269)$ & $4.0(21 / 518)$ & $1.42(0.72-2.80)$ & $1.51(0.72-3.16)$ & 0.28 \\
\hline $11-15$ & $10.0(27 / 269)$ & $9.5(49 / 518)$ & $1.01(0.60-1.69)$ & $1.02(0.60-1.76)$ & 0.93 \\
\hline $16-20$ & $7.1(19 / 269)$ & $9.3(48 / 518)$ & $0.68(0.37-1.24)$ & $0.69(0.36-1.32)$ & 0.26 \\
\hline$>20$ & $11.2(30 / 269)$ & $16.8(87 / 518)$ & $0.57(0.36-0.93)$ & $0.62(0.38-1.03)$ & 0.07 \\
\hline Test of trend & & & & & $p=0.09$ \\
\hline Unspecified & $4.8(13 / 271)$ & $3.5(18 / 518)$ & & & \\
\hline \multicolumn{6}{|c|}{ Infectious mononucleosis } \\
\hline Never & $70.9(183 / 258)$ & $82.7(420 / 508)$ & 1.00 (Reference) & 1.00 (Reference) & \\
\hline $6-10$ & $1.2(3 / 258)$ & $1.0(5 / 508)$ & $1.38(0.30-6.45)$ & $1.75(0.31-9.84)$ & 0.53 \\
\hline $11-15$ & $4.7(12 / 258)$ & $2.6(13 / 508)$ & $2.20(0.92-5.24)$ & $2.84(1.07-7.56)$ & 0.04 \\
\hline $16-20$ & $12.4(32 / 258)$ & $7.5(38 / 508)$ & $1.91(1.15-3.18)$ & $1.94(1.14-3.31)$ & 0.02 \\
\hline$>20$ & $8.5(22 / 258)$ & $5.1(26 / 508)$ & $1.83(1.02-3.31)$ & $1.86(1.00-3.44)$ & 0.05 \\
\hline Test of trend & & & & & $p=0.001^{\dagger}$ \\
\hline Unspecified & $2.3(6 / 258)$ & $1.2(6 / 508)$ & & & \\
\hline \multicolumn{6}{|c|}{ Impetigo } \\
\hline Never & $91.9(238 / 259)$ & $91.5(462 / 505)$ & 1.00 (Reference) & 1.00 (Reference) & \\
\hline $0-5$ & $0.0(0 / 259)$ & $0.0(0 / 505)$ & - & - & \\
\hline $6-10$ & $5.2(13 / 259)$ & $5.2(26 / 505)$ & $1.08(0.53-2.23)$ & $0.99(0.47-2.10)$ & 0.99 \\
\hline $11-15$ & $1.5(4 / 259)$ & $2.4(12 / 505)$ & $0.69(0.21-2.21)$ & $0.69(0.21-2.28)$ & 0.54 \\
\hline $16-20$ & $0.0(0 / 259)$ & $0.0(0 / 505)$ & - & - & \\
\hline$>20$ & $0.0(0 / 259)$ & $0.0(0 / 505)$ & - & - & \\
\hline Test of trend & & & $p=0.78^{\dagger}$ & $p=0.66^{\dagger}$ & \\
\hline Unspecified & $1.5(4 / 259)$ & $1.0(5 / 505)$ & & & \\
\hline
\end{tabular}

CNS: central nervous system demyelination, OR: odds ratio, CI: confidence interval

* Odds ratio adjusted for highest level of education, smoking history and serum 25-hydroxyvitamin D (25OHD) level

$*$ Positive history of infection or tonsillectomy, but age at diagnosis not specified

${ }^{\dagger} P$ value for a homogeneity test by which we test for a differential effect of infection at different ages within the age span $0-20+$ years 
Our study had a number of limitations. The vaccination, infection and tonsillectomy data were self-reported and retrospective. However, in analysis including only those cases who thought infection was not an important cause of their illness and their matched controls, our findings were very similar to those for the full group, suggesting there was not differential recall based on a belief about infection as a risk factor for the illness. Furthermore, the frequency of 'unspecified' (i.e. missing age group data) responses did not differ between cases and controls. A reliability study comparing self-reported age at measles infection to medical records showed $86 \%$ sensitivity for 'measles infection between 5 and 9 years of age', but inaccuracy for actual age (in years) that did not differ in direction or magnitude between cases and controls (Haile et al., 1982). Such non-differential misclassification will bias odds ratio estimates towards unity, potentially underestimating effect sizes for any age effects.

Our study was limited by the sometimes small number of participants who did not receive a vaccination (e.g. against poliomyelitis or diphtheria), or who did have a specific infection. We did not have information on the specific vaccine that was administered, e.g. live attenuated vs. inactivated for poliomyelitis, and different participants would have been exposed to changing childhood vaccination schedules over time, e.g. moving from a measles-mumps vaccine (introduced in 1982) to the MMR vaccine (introduced in 1989) (Burgess, 2003). Nevertheless, our cases and controls were matched on age, and casecontrol sets would thus have been exposed to the same vaccination schedules. We report a large number of analyses; thus it is possible that some results with a $p$-value of $<0.05$ are due to chance alone. However, we note that the smallest $p$-value for the well-accepted association between history of IM and risk of CNS demyelination in our age group analyses was 0.02 (for the 16-20 years age group, with that for age 11-15 years, $p=0.04$ ). We believe that results returning $p$-values around this level should be seriously considered as potentially causally related to the risk of a first clinical diagnosis of CNS demyelination.

A strength of the study is that the questionnaire seeking information on past vaccinations and infections was mailed to participants, to complete with reference to any vaccination or medical records, and potentially with additional recall by a parent. Other strengths of this study are the population-based setting and ascertainment of incident cases with a first clinical diagnosis of CNS demyelination. In the control participants, 76.3\% self-reported 'ever-vaccinated' against rubella; this is very similar to the $75.5 \%$ in a national report on childhood immunisation (ABS. Children's Immunisation Australia 1996).

In a recent meta-analysis, a history of tonsillectomy prior to age 20 years was associated with an increased risk of MS (pooled OR $=1.32$, $95 \%$ CI 1.08-1.61, $p=0.006 ; 12$ studies, $\mathrm{I}^{2}=44 \%$ ), consistent with our findings for a first clinical diagnosis of CNS demyelinatio (Lunny et al., 2013). This effect was not apparent for tonsillectomy after 20 years of age (pooled OR $=1.20,95 \%$ CI $0.94-1.53, p=0.15 ; 9$ studies, $\mathrm{I}^{2}=32 \%$ ). Tonsillectomy may represent an easily recalled marker of recurrent tonsillitis, possibly a result of colonisation with Epstein Barr Virus (EBV) (Dias et al., 2009). There are few studies testing the role of EBV infection in recurrent tonsillitis. One study showed that IM was somewhat less common in those who had had a tonsillectomy (Sumaya et al., 1978); we found no association between IM and tonsillectomy in control participants in the current study.

Consistent with our findings, a recent meta-analysis (Farez and Correale, 2011) found no evidence of an association with MS risk for prior vaccination for measles $(\mathrm{OR}=1.07,95 \% \mathrm{CI} 0.97-1.19,6$ studies, $I^{2}=4.1 \%$ ), mumps (OR $=1.09$, 95\%CI 1.02-1.18, 4 studies, $I^{2}=0.0 \%$ ), or tuberculosis (OR $=0.96,95 \%$ CI $0.69-1.34,6$ studies, $I^{2}=0.0 \%$ ), with insufficient evidence to evaluate pertussis vaccination. For rubella vaccination, the evidence was conflicting with high heterogeneity between studies $\left(I^{2}=84.6 \%, p=0.0001\right.$ ) (Farez and Correale, 2011). Our finding of increased risk of a first clinical diagnosis of CNS demyelination in association with poliomyelitis vaccination is at odds with the null findings of the meta-analysis (OR $=0.87,95 \% \mathrm{CI}$ $0.61-1.25$, 7 studies, $\mathrm{I}^{2}=28 \%$ ), but the latter did not examine agespecific effects. Also in contrast to our results, the meta-analysis showed a reduced risk of MS in association with vaccination for diphtheria $\left(\mathrm{OR}=0.60,95 \% \mathrm{CI} 0.40-0.90,3\right.$ studies, $\left.\mathrm{I}^{2}=0.0 \%\right)$ and tetanus $\left(\mathrm{OR}=0.68,95 \% \mathrm{CI} 0.54-0.84,8\right.$ studies, $\left.\mathrm{I}^{2}=8.3 \%\right)$ (Farez and Correale, 2011). As well as age effects, the discrepant findings between studies may be related to differences in vaccines and schedules over time, for example, use of inactivated vs. live attenuated polio virus and monovalent vs. combined diphtheria-pertussis-tetanus vaccines.

Studies examining age at vaccination and MS risk are rare. In a population-based case-control study in Sweden (Ahlgren et al., 2009), having been vaccinated against measles, mumps or rubella did not influence MS risk but, compared to MMR vaccination given at other ages, early MMR vaccination ( $\leq 10$ years) was associated with an increased risk of MS (OR $=4.92$, 95\%CI 1.97-12.20, $p=0.0006$ ) (and, conversely, late $\mathrm{MMR}$ vaccination with a reduced risk $\mathrm{OR}=0.40,95 \% \mathrm{CI}$ 0.17-0.93). Most other studies consider vaccination in relation to time of diagnosis, e.g. previous 5 years, rather than age at vaccination (DeStefano et al., 2003, Hernan et al., 2006, Farez and Correale, 2011, Zilber and Kahana, 1996, Lucas et al., 2007, Lucas et al., 2011, Rothman, 1990, Greenland et al., 2016, Haile et al., 1982, Burgess, 2003, ABS. Children's Immunisation Australia 1996, Lunny et al., 2013, Dias et al., 2009, Sumaya et al., 1978, Hernan et al., 2004). In a recent large case-control study using administrative data, 'any vaccination' in the previous 5 years was associated with a reduced risk of developing MS, particularly vaccination for influenza or tickborne encephalitis (Hapfelmeier et al., 2019). Vaccinations specifically in childhood were not considered.

If immunisation against a pathogen reduces the risk of CNS demyelination, it might be expected that infection with that pathogen would be associated with increased risk. Our finding of a halving of risk of a first clinical diagnosis of CNS demyelination in association with

Table 5

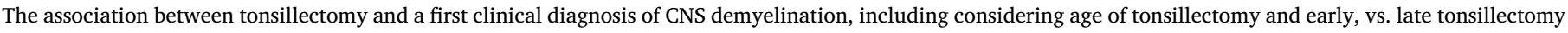

\begin{tabular}{|c|c|c|c|c|c|}
\hline Tonsillectomy (years) & Cases $\%(n / N)$ & Controls \% $(n / N)$ & OR $(95 \% \mathrm{CI})$ & $\mathrm{AOR}^{*}(95 \% \mathrm{CI})$ & $\mathrm{p}$ \\
\hline Never & $69.3(190 / 274)$ & $75.0(396 / 528)$ & 1.00 (Reference) & 1.00 (Reference) & \\
\hline $0-5$ & $9.5(26 / 274)$ & $9.1(48 / 528)$ & $1.17(0.69-1.99)$ & $1.22(0.70-2.13)$ & 0.48 \\
\hline $6-10$ & $9.9(27 / 274)$ & $6.8(36 / 528)$ & $1.74(1.01-3.01)$ & $1.77(0.99-3.14)$ & 0.05 \\
\hline $11-15$ & $5.8(16 / 274)$ & $3.4(18 / 528)$ & $2.08(0.98-4.40)$ & $2.45(1.12-5.35)$ & 0.03 \\
\hline $16-20$ & $1.8(5 / 274)$ & $2.1(11 / 528)$ & $1.33(0.42-4.16)$ & $0.82(0.19-3.50)$ & 0.79 \\
\hline$>20$ & $3.3(9 / 274)$ & $3.0(16 / 528)$ & $1.17(0.51-2.71)$ & $1.12(0.47-2.68)$ & 0.79 \\
\hline Test of trend & & & & & $p=0.11 \uparrow$ \\
\hline Unspecified + & $0.4(1 / 274)$ & $0.6(3 / 528)$ & & & \\
\hline Tonsillectomy versus no tonsillectomy & $30.7(84 / 274)$ & $25.0(132 / 528)$ & $1.42(1.00-2.02)$ & $1.46(1.01-2.12)$ & 0.05 \\
\hline Early $^{\mathrm{a}}$ tonsillectomy versus no tonsillectomy & $21.8(53 / 243)$ & $17.5(84 / 480)$ & $1.44(0.94-2.22)$ & $1.54(0.97-2.43)$ & 0.07 \\
\hline Late $^{\mathrm{b}}$ tonsillectomy versus no tonsillectomy & $13.6(30 / 220)$ & $10.2(45 / 441)$ & $1.31(0.98-1.76)$ & $1.28(0.94-1.75)$ & 0.11 \\
\hline Late $^{\mathrm{b}}$ tonsillectomy versus early ${ }^{\mathrm{a}}$ tonsillectomy & $36.1(30 / 83)$ & $34.9(45 / 129)$ & $1.05(0.50-2.22)$ & $1.20(0.53-2.72)$ & 0.66 \\
\hline
\end{tabular}

* Odds ratio adjusted for highest level of education, smoking history and serum 25-hydroxyvitamin D (25OHD) level 
late rubella vaccination (11-15 years compared to never) that was independent of history of rubella infection, along with a finding of a 3fold increase in risk of a first clinical diagnosis of CNS demyelination for late rubella infection (11-15 years compared to never) provides some confidence that this may be a true association. Nevertheless, previous studies have returned inconsistent findings in relation to MS risk and late rubella infection (Compston et al., 1986, Bachmann and Kesselring, 1998, Hernan et al., 2001, Zorzon et al., 2003, Casetta et al., 1994, Pekmezovic et al., 2004, Zaadstra et al., 2008). A challenge with self-reported rubella infection, is that it is typically a relatively mild infection that can be misdiagnosed. In a reliability study (Ahlgren et al., 2009) where self-reported infection history was validated by child and school health records, there was $54 \%$ and $47 \%$ sensitivity (MS cases and controls, respectively) for rubella compared to $72 \%$ and $64 \%$ for varicella, $72 \%$ and $68 \%$ for mumps, and $83 \%$ and $79 \%$ for measles.

We found no evidence that infection with varicella, measles, mumps, pertussis, genital herpes or impetigo, whether acquired at an early or late age, were associated with a first clinical diagnosis of CNS demyelination. This is largely consistent with previous studies, including in relation to age at infection (Zorzon et al., 2003, Zaadstra et al., 2008, Marrie et al., 2000). Some studies have reported an increased risk of MS in association with older age at measles infection, (Gronning et al., 1993, Compston et al., 1986, Bachmann and Kesselring, 1998, Sullivan et al., 1984) but this has not been consistently found (Hernan et al., 2001, Zorzon et al., 2003, Casetta et al., 1994, Pekmezovic et al., 2004, Zaadstra et al., 2008). Similarly, some previous studies (Bachmann and Kesselring, 1998, Hays, 1992, Hernan et al., 2001) demonstrate a positive association between late infection with mumps and MS but others do not (Haile et al., 1982, Pekmezovic et al., 2004, Zaadstra et al., 2008).

Our results showed a significant trend of increasing risk of a first clinical diagnosis of CNS demyelination with older reported age of IM. This is consistent with some (Hernan et al., 2001, Marrie et al., 2000), but not all (Zorzon et al., 2003, Casetta et al., 1994, Zaadstra et al., 2008), previous studies of IM and risk of MS. Consistent with a previous large population-based case-control study, we found no significant effect on MS risk of a second infection in combination with a history of IM (Ahlgren et al., 2009).

In summary, we found an increased risk of a first diagnosis of CNS demyelination in association with poliomyelitis vaccination prior to school age, and a reduced risk with older age at rubella vaccination. For infections, the 11-15 years age group seemed particularly important with increased risk of a first clinical diagnosis of CNS demyelination in association with history of rubella and/or infectious mononucleosis. Our findings suggest a difference between cases with a first clinical diagnosis of CNS demyelination and population controls with respect to environment in adolescence and early adulthood and are coherent with the idea of MS as an age-dependent response to viral, particularly EBV, infection.

\section{CRediT authorship contribution statement}

A.M. Hughes: Conceptualization, Data curation, Formal analysis, Writing - original draft, Writing - review \& editing. A.-L. Ponsonby: Funding acquisition, Investigation, Project administration, Resources, Writing - review \& editing. K. Dear: Formal analysis, Funding acquisition, Investigation, Methodology, Project administration, Resources, Writing - review \& editing. T. Dwyer: Funding acquisition, Investigation, Methodology, Project administration, Resources, Writing - review \& editing. B.V. Taylor: Funding acquisition, Investigation, Methodology, Project administration, Resources, Writing - review \& editing. I. van der Mei: Funding acquisition, Investigation, Methodology, Project administration, Resources, Writing - review \& editing. P.C. Valery: Funding acquisition, Investigation, Methodology, Project administration, Resources, Writing - review \& editing. R.M. Lucas: Conceptualization, Data curation, Formal analysis,
Investigation, Methodology, Project administration, Resources, Supervision, Visualization, Writing - review \& editing.

\section{Declaration of Competing Interest}

The authors declare no conflicts of interest

\section{Acknowledgments}

We would like to acknowledge and thank the physicians who notified case participants to the Ausimmune Study:

Ioanne Anderson, FRANZCO, Coastal Eye Centre Queensland; Michael Bailey, FRANZCO, The Mount Gambier Eye Centre South Australia; Peter Batchelor, PhD, Barwon Health, Victoria; Jeffrey Blackie, FRACP, John Hunter Hospital, Newcastle, New South Wales; Richard Bourke, FRACGP, General Practice, Tasmania; Richard Boyle, FRACP, Princess Alexandra Hospital, Brisbane, Queensland; John Cameron, MD, Princess Alexandra Hospital, Brisbane, Queensland; Ross Carne, MD, Deakin University, Victoria; Chris Charnley, FRACP, Southwest Healthcare, Warrnambool, Victoria; Ben Clark, FRANZCO, Geelong Hospital, Victoria; Steven Collins, MD, St Vincent's Hospital, Melbourne; Diana Conrad, FRANZCO, Wesley Medical Centre, Auchenflower, Queensland; Michael Coroneos, FRACS, Private Practice, Brisbane, Queensland; Nicholas Downie, FRANZCO, Launceston General Hospital, Tasmania; Michael Dreyer, MD, Royal Hobart Hospital, Tasmania; Mervyn Eadie, MD, Royal Brisbane and Women's Hospital, Queensland; David Floate, FRACP, John Hunter Hospital, Newcastle, New South Wales; Peter Gates, FRACP, Barwon Health, Geelong Hospital, Victoria; Kerryn Green, FRACP, University of Queensland, Queensland; Erwin Groeneveld, FRANZCO, Princess Alexandra Hospital, Brisbane, Queensland; Mark Guirguis, MBBS (Hons), Private Practice, Tasmania; John Harrison, FRANZCO, Royal Brisbane and Women's Hospital and Princess Alexandra Hospital, Brisbane, Queensland; Michael Haybittel, FRANZCO, North West Regional Hospital, Tasmania; Robert Henderson, FRACP, Royal Brisbane and Women's Hospital, Queensland; John Henshaw, MMed, University of Tasmania, Tasmania; Keith Ho, MBBS, Ballarat Medical Centre, Victoria; Eugene Hollenbach, MBBS, Private Practice, Newcastle, New South Wales; James Hurley, MD, University of Melbourne, Victoria; Dean Jones, FRACP, Royal Hobart Hospital, Tasmania; Michael Katekar, MBBS, John Hunter Hospital, Newcastle, New South Wales; Anthony Kemp, FRACP, Ballarat Health Services, Victoria; Mark King, FRACP, Geelong Private Hospital, Victoria; George Kiroff, FRACS, The Geelong Hospital, Victoria; Brett Knight, FRACP, Ballarat Health Services, Victoria; Thomas Kraemer, FRACP, The Geelong Hospital, Victoria; Cecile Lander, FRACP, Royal Brisbane and Women's Hospital, Queensland; Jeanette Lechner-Scott, FRACP, John Hunter Hospital, Newcastle, New South Wales; Patrick Lockie, FRACS, St John of God Hospital, Geelong, Victoria; Andre Loiselle, FRACP, Hunter New England Health, Newcastle, New South Wales; Paul McCartney, FRANZCO, Royal Hobart Hospital, Tasmania; Pam McCombe, PhD, University of Queensland, Queensland; Mark McGree, FRANZCO, McCullough Medical Centre, Queensland; David McKnight, FRANZCO, Ballarat Base Hospital, Victoria; Dan McLaughlin, PhD, Royal Brisbane and Women's Hospital, Queensland; Ian Murrell, FRANZCO, The Eye Hospital, Launceston, Tasmania; Satish Nagarajah, MBBS, St John of God Hospital, Geelong, Victoria; Robert Newton, MBBS, Bayside Medical Centre, Hobart, Tasmania; Rob Nightingale, FRACP, Calvary Hospital, Tasmania; Terence O'Brien, MD, University of Melbourne, Victoria; John O'Sullivan, MD, Royal Brisbane and Women's Hospital, Queensland; Gregory Outteridge, FRANZCO, Hunter Valley Private Hospital, Newcastle, New South Wales; Anthony Pane, FRANZCO, Queensland Eye Institute, Queensland; Mark Parsons, FRACP, Hunter Medical Research Institute, Newcastle, New South Wales; Melinda Pascoe, FRACP, Private Practice, Brisbane, Queensland; 
David Prentice, PhD FRACP, St Vincent's Hospital, Melbourne, Victoria; Richard Ralph, FRACGP, Cascade road Medical Centre, Hobart, Tasmania; Stephen Read, FRACP, Royal Brisbane and Women's Hospital, Queensland; Alison Reid, FRACP, Private Practice, Brisbane, Queensland; John Richmond, FRACP, Ballarat Health Services, Victoria; Ian Routley, FRANZCO, The Geelong Hospital, Victoria; Timothy Ruddell, FRANZCO, Private Practice, Newcastle, New South Wales; Noel Saines, FRACP, Wesley Medical Centre, Auchenflower, Queensland; Stan Siejka (dec), MBBS, Launceston General Hospital, Tasmania; Peter Silburn, PhD FRACP, University of Queensland, Queensland; Christopher Staples, FRACP, Mater Health Services, Brisbane, Queensland; Alice Ann Sullivan, FRACP, Royal Brisbane and Women's Hospital, Brisbane, Queensland; Paul Talman, FRACP, Barwon Health, Geelong Hospital, Victoria; Don Todman, FRACP, University of Queensland, Queensland; Nitin Verma, FRANZCO, Hobart Eye Surgeons, Tasmania; Brendan Vote, FRANZCO, University of Tasmania, Tasmania; Michael Waldie, FRANZCO, Queensland Eye Hospital, Queensland; Michael Weetch, FRACP, Private Practice, Bendigo, Victoria; Rodney Westmore, FRANZCO, Launceston General Hospital, Tasmania; Andrew Wong, FRACP, Princess Alexandra Hospital, Brisbane, Queensland. Notifying physicians received no payment for case notification to the study.

We would like to acknowledge the outstanding input of the paid research personnel, including the local research officers:

Susan Agland, BN, Hunter New England Health, Newcastle, New South Wales; Barbara Alexander, BN, Queensland Institute for Medical Research, Queensland; Marcia Davis, MD, Queensland Institute for Medical Research, Queensland; Zoe Dunlop, BN, Barwon Health, Geelong Hospital, Victoria; Rosalie Scott, BN, Royal Brisbane and Women's Hospital, Queensland; Marie Steele, RN, Royal Brisbane and Women's Hospital, Queensland; Catherine Turner, MPH\&TM, Menzies Research Institute, Tasmania; Brenda Wood, RN, Menzies Research Institute, Tasmania; and the Ausimmune Study project officers during the course of the study:

Jane Gresham, MA (Int Law), National Centre for Epidemiology and Population Health, The Australian National University, Canberra; Australian Capital Territory; Camilla Jozwick, BSc(Hons), National Centre for Epidemiology and Population Health, The Australian National University, Canberra; Australian Capital Territory; Helen Rodgers, RN, National Centre for Epidemiology and Population Health, The Australian National University, Canberra; Australian Capital Territory.

\section{Funding}

The Ausimmune Study was funded by the National Multiple Sclerosis Society of the USA (RG3364A1/2). Patricia Valery was supported by a NHMRC Career Development Fellowship (1083090); Robyn M Lucas (1107343) and Anne-Louise Ponsonby (1110200) are supported by NHMRC Senior Career Fellowships.

\section{Supplementary materials}

Supplementary material associated with this article can be found, in the online version, at doi:10.1016/j.msard.2020.102062.

\section{References}

Abbasi, M, Nabavi, S.M., Fereshtehnejad, S.M., et al., 2017. Multiple sclerosis and environmental risk factors: a case-control study in Iran. Neurol. Sci. 38, 1941-1951.
ABS. Children's Immunisation Australia. Canberra: Australian bureau of statistics, 1996. Ahlgren, C, Toren, K, Oden, A, Andersen, O, 2009. A population-based case-control study on viral infections and vaccinations and subsequent multiple sclerosis risk. Eur. J. Epidemiol. 24, 541-552.

Ascherio, A, Munger, KL, 2007. Environmental risk factors for multiple sclerosis. Part II: Noninfectious factors. Ann. Neurol. 61, 504-513.

Bachmann, S, Kesselring, J, 1998. Multiple sclerosis and infectious childhood diseases. Neuroepidemiology 17, 154-160.

Bager, P, Nielsen, N.M., Bihrmann, K, et al., 2004. Childhood infections and risk of multiple sclerosis. Brain 127, 2491-2497.

Burgess, M, 2003. Immunisation: a public health success. NSW Public Health Bulletin 14.

Casetta, I, Granieri, E, Malagu, S, et al., 1994. Environmental risk factors and multiple sclerosis: a community-based, case-control study in the province of Ferrara, Italy. Neuroepidemiology 13, 120-128.

Compston, D.A., Vakarelis, B.N., Paul, E, McDonald, W.I., Batchelor, J.R., Mims, CA, 1986. Viral infection in patients with multiple sclerosis and HLA-DR matched controls. Brain 109 (Pt 2), 325-344.

DeStefano, F, Verstraeten, T, Jackson, LA, et al., 2003. Vaccinations and risk of central nervous system demyelinating diseases in adults. Arch. Neurol. 60, 504-509.

Dias, E.P., Rocha, M.L., Carvalho, M.O., Amorim, L.M., 2009. Detection of Epstein-Barr virus in recurrent tonsillitis. Braz. J. Otorhinolaryngol. 75, 30-34.

Farez, M.F., Correale, J, 2011. Immunizations and risk of multiple sclerosis: systematic review and meta-analysis. J. Neurol. 258, 1197-1206.

Greenland, S, Senn, S.J., Rothman, K.J., et al., 2016. Statistical tests, $p$ values, confidence intervals, and power: a guide to misinterpretations. Eur. J. Epidemiol. 31, 337-350.

Gronning, M, Riise, T, Kvale, G, Albrektsen, G, Midgard, R, Nyland, H, 1993. Infections in childhood and adolescence in multiple sclerosis. A case-control study. Neuroepidemiology 12, 61-69.

Haile, R, Smith, P, Read, D, Nassim, D, Warlow, C, Russell, W.C., 1982. A study of measles virus and canine distemper virus antibodies, and of childhood infections in multiple sclerosis patients and controls. J. Neurol. Sci. 56, 1-10.

Hapfelmeier, A, Gasperi, C, Donnachie, E, Hemmer, B, 2019. A large case-control study on vaccination as risk factor for multiple sclerosis. Neurology 93, e908-ee16.

Hays, P, 1992. Multiple sclerosis and delayed mumps. Acta Neurol. Scand. 85, 200-203.

Hernan, M.A., Zhang, S.M., Lipworth, L, Olek, M.J., Ascherio, A, 2001. Multiple sclerosis and age at infection with common viruses. Epidemiology 12, 301-306.

Hernan, M.A., Jick, S.S., Olek, M.J., Jick, H, 2004. Recombinant hepatitis B vaccine and the risk of multiple sclerosis: a prospective study. Neurology 63, 838-842.

Hernan, M.A., Alonso, A., Hernandez-Diaz, S, 2006. Tetanus vaccination and risk of multiple sclerosis: a systematic review. Neurology 67, 212-215.

International Multiple Sclerosis Genetics Consortium, 2018. Low-frequency and rarecoding variation contributes to multiple sclerosis risk. Cell 175 1679-87 e7.

Lucas, R, Ponsonby, A.L., McMichael, A, et al., 2007. Observational analytic studies in multiple sclerosis: controlling bias through study design and conduct. The Australian multicentre study of environment and immune function. Mult. Scler. 13, 827-839.

Lucas, R.M., Ponsonby, A.L., Dear, K, et al., 2011. Sun exposure and vitamin D are independent risk factors for CNS demyelination. Neurology 76, 540-548.

Lunny, C, Knopp-Sihota, J.A., Fraser, S.N., 2013. Surgery and risk for multiple sclerosis: a systematic review and meta-analysis of case-control studies. BMC Neurol. 13, 41.

Mailand, M.T., Frederiksen, J.L., 2017. Vaccines and multiple sclerosis: a systematic review. J. Neurol. 264, 1035-1050.

Marrie, R.A., Wolfson, C, Sturkenboom, M.C., et al., 2000. Multiple sclerosis and antecedent infections: a case-control study. Neurology 54, 2307-2310.

Pekmezovic, T, Jarebinski, M, Drulovic, J, 2004. Childhood infections as risk factors for multiple sclerosis: belgrade case-control study. Neuroepidemiology 23, 285-288.

Ribbons, K, Lea, R, Tiedeman, C, Mackenzie, L, Lechner-Scott, J, 2017. Ongoing increase in incidence and prevalence of multiple sclerosis in Newcastle, Australia: a 50-year study. Mult. Scler. 23, 1063-1071.

Rothman, K.J., 1990. No adjustments are needed for multiple comparisons. Epidemiology $1,43-46$.

Shaygannejad, V, Rezaie, N, Paknahad, Z, Ashtari, F, Maghzi, H, 2016. The environmental risk factors in multiple sclerosis susceptibility: a case-control study. Adv. Biomed. Res. 5, 98.

Sullivan, C.B., Visscher, B.R., Detels, R, 1984. Multiple sclerosis and age at exposure to childhood diseases and animals: cases and their friends. Neurology 34, 1144-1148.

Sumaya, C.V., Downey, K, Ullis, K.C., 1978. Tonsillectomy and infectious mononucleosis. Am. J. Epidemiol. 107, 65-70.

Waubant, E, Lucas, R, Mowry, E, et al., 2019. Environmental and genetic risk factors for MS: an integrated review. Ann. Clin. Transl. Neurol.

Wendel-Haga, M, Celius, E.G., 2017. Is the hygiene hypothesis relevant for the risk of multiple sclerosis? Acta Neurol. Scand. 201, 26-30 136 Suppl.

Zaadstra, B.M., Chorus, A.M., van Buuren, S, Kalsbeek, H, van Noort, J.M., 2008. Selective association of multiple sclerosis with infectious mononucleosis. Mult. Scler. 14, 307-313.

Zilber, N, Kahana, E, 1996. Risk factors for multiple sclerosis: a case-control study in Israel. Acta Neurol. Scand. 94, 395-403.

Zorzon, M, Zivadinov, R, Nasuelli, D, et al., 2003. Risk factors of multiple sclerosis: a casecontrol study. Neurol. Sci. 24, 242-247. 